\title{
Adaptive Control for Linear Uncertain Systems with Unmodeled Dynamics Revisited via Optimal Control Modification
}

\author{
Nhan Nguyen* \\ NASA Ames Research Center, Moffett Field, CA 94035
}

\begin{abstract}
This paper presents the optimal control modification for linear uncertain plants. The Lyapunov analysis shows that the modification parameter has a limiting value depending on the nature of the uncertainty. The optimal control modification exhibits a linear asymptotic property that enables it to be analyzed in a linear time invariant framework for linear uncertain plants. The linear asymptotic property shows that the closedloop plants in the limit possess a scaled input-output mapping. Using this property, we can derive an analytical closed-loop transfer function in the limit as the adaptive gain tends to infinity. The paper revisits the Rohrs counterexample problem that illustrates the nature of non-robustness of model-reference adaptive control in the presence of unmodeled dynamics. An analytical approach is developed to compute exactly the modification parameter for the optimal control modification that stabilizes the plant in the Rohrs counterexample. The linear asymptotic property is also used to address output feedback adaptive control for non-minimum phase plants with a relative degree 1.
\end{abstract}

\section{Introduction}

Research in robust adaptive control was motivated by instability phenomena of adaptive control. In fact, instability of adaptive control in the early 1960's which contributed to the crash of one of the NASA X-15 hypersonic vehicles caused a great deal of concern about the viability of adaptive control. The standard model-reference adaptive control is well-known to be non-robust in the presence of time delay, unmodeled dynamics, and disturbances. Rohrs et al. investigated instability mechanisms of adaptive control due to unmodeled dynamics in the 1980's. ${ }^{1}$ Thus, the field of adaptive control has seen numerous robust modification techniques developed to improve robustness. As a result, various robust modification schemes had since been developed to ensure boundedness of adaptive parameters. The $\sigma$ modification $^{2}$ and $e$ modification ${ }^{3}$ are two well-known robust modification methods. Other techniques such as the deadband and projection methods are also used to improve robustness of adaptive control algorithms. In recent years, there have been various new advanced robust adaptive control methods being developed such as the $\mathscr{L}_{1}$ method, ${ }^{4}$ optimal control modification, ${ }^{5,6}$ adaptive loop recovery, ${ }^{7}$ composite model reference adaptive control, ${ }^{8}$ and many others. The principle of robust modification is based on two central themes: 1) limiting adaptive parameters and 2) adding damping mechanisms to model-reference adaptive control. The robustness issues with parameter drift, nonminimum phase behaviors, time delay, unmodeled dynamics, and fast adaptation are largely ameliorated with these robust modification schemes, but are not entirely eliminated if the nature of the uncertainty is not completely known.

Robust adaptive control achieves robustness by compromising the ideal property of asymptotic tracking of MRAC. All robust modifications in adaptive control result in bounded tracking and adaptive parameters when they are properly designed. Recognizing this important requirement of robust adaptive control, the optimal control modification is a recent development of robust modification in adaptive control that addresses adaptive control in the framework of optimal control. More specifically, the optimal control modification is designed to minimize the tracking error norm bounded away from the origin by some lower bound.

In this paper, we revisit the Rohrs counterexample problem that illustrates the nature of non-robustness of MRAC in the presence of unmodeled dynamics and develop an analytical approach that computes exactly the modification parameter using the optimal control modification to stabilize the plant in the Rohrs counterexample. The optimal control modification exhibits a linear asymptotic property that enables it to be analyzed in a linear time invariant framework when the uncertain plant is also linear time invariant. Using this property, we can derive an analytical

${ }^{*}$ Research Scientist, Intelligent Systems Division, AIAA Associate Fellow, nhan.t.nguyen@nasa.gov 
closed-loop transfer function in the limit as the adaptive gain tends to infinity. In contrast, both the $\sigma$ modification and $e$ modification can stabilize the Rohrs plant as well, but the modification parameters are chosen ad-hoc.

\section{Optimal Control Modification for Linear Time Invariant Uncertain Systems}

Consider the following linear time invariant system with matched uncertainty

$$
\dot{x}=A x+B\left(u+\Theta^{* \top} x\right)+w
$$

where $x(t) \in \mathbb{R}^{n}$ is a state vector, $u(t) \in \mathbb{R}^{m}$ is a control vector, $A \in \mathbb{R}^{n} \times \mathbb{R}^{n}$ is known, $B \in \mathbb{R}^{n \times m}$ is also known such that $(A, B)$ is controllable, $\Theta^{*} \in \mathbb{R}^{n \times m}$ is the unknown parameter constant matrix, where $w$ is a unknown bounded disturbance

The reference model is given by

$$
\dot{x}_{m}=A_{m} x_{m}+B_{m} r
$$

where $A_{m} \in \mathbb{R}^{n} \times \mathbb{R}^{n}$ is known and Hurwitz, $B_{m} \in \mathbb{R}^{n} \times \mathbb{R}^{r}$ is known, and $r(t) \in \mathbb{R}^{r}$ is a bounded reference command signal.

Assuming that there exist matching model conditions

$$
\begin{gathered}
A+B K_{x}=A_{m} \\
B K_{r}=B_{m}
\end{gathered}
$$

Then adaptive controller is designed as

$$
u=K_{x} x+K_{r} r-\Theta^{\top} x
$$

Defining the tracking error as $e=x_{m}-x$, then the error equation is obtained as

$$
\dot{e}=A_{m} e+B \tilde{\Theta}^{\top} \Phi(x)-w
$$

The adaptive parameter $\Theta$ can be estimated using the optimal control modification adaptive law as

$$
\dot{\Theta}=-\Gamma x\left(e^{\top} P-v x^{\top} \Theta B^{\top} P A_{m}^{-1}\right) B
$$

where $\Gamma=\Gamma^{\top}>0 \in \mathbb{R}^{n \times n}$ is a positive-definite adaptive gain matrix, $v>0 \in \mathbb{R}$ is the optimal control modification parameter, and $P=P^{\top}>0 \in \mathbb{R}^{n \times n}$ is a positive-definite matrix that solve the following Lyapunov equation

$$
P A_{m}+A_{m}^{\top} P=-Q
$$

where $Q=Q^{\top}>0 \in \mathbb{R}^{n \times n}$ is a positive-definite weighting matrix.

Theorem 1: The optimal control modification adaptive law is stable for any modification parameter $0<v<v_{\max }$ where

$$
v_{\text {max }}=\frac{\lambda_{\min }(Q) \lambda_{\text {min }}\left(B^{\top} A_{m}^{-\top} Q A_{m}^{-1} B\right)}{\left\|B^{\top} P A_{m}^{-1} B\right\|^{2} \Theta_{0}^{2}}
$$

with $\Theta_{0}=\max \left\|\Theta^{*}\right\|$ as the upper bound on the parametric uncertainty.

Moreover, if $\Theta$ is a non-destabilizing parametric uncertainty, then

$$
v_{\text {max }}=\frac{4 \lambda_{\min }\left(B^{\top} A_{m}^{-\top} Q A_{m}^{-1} B\right)\|P B\|^{2}}{\lambda_{\text {min }}(Q)\left\|B^{\top} P A_{m}^{-1} B\right\|^{2}}
$$

independent of the upper bound on the parametric uncertainty $\Theta_{0}$.

Proof: Choose a Lyapunov candidate function

$$
V(e, \tilde{\Theta})=e^{\top} P e+\operatorname{trace}\left(\tilde{\Theta}^{\top} \Gamma^{-1} \tilde{\Theta}\right)
$$

Differentiating $V(e, \tilde{\Theta})$ yields

$$
\begin{aligned}
\dot{V}(e, \tilde{\Theta})=-e^{\top} Q e+2 e^{\top} P B \tilde{\Theta}^{\top} x-2 e^{\top} P w-2 \operatorname{trace}\left[\tilde{\Theta}^{\top} x\left(e^{\top} P-v x^{\top} \Theta B^{\top} P A_{m}^{-1}\right) B\right] \\
=-e^{\top} Q e-2 e^{\top} P w+2 v x^{\top} \tilde{\Theta} B^{\top} P A_{m}^{-1} B \tilde{\Theta}^{\top} x+2 v x^{\top} \Theta^{*} B^{\top} P A_{m}^{-1} B \tilde{\Theta}^{\top} x
\end{aligned}
$$


$P A_{m}^{-1}$ can be decomposed into a symmetric part $M$ and anti-symmetric part $N$ as

$$
P A_{m}^{-1}=M+N
$$

where

$$
\begin{gathered}
M=\frac{1}{2}\left(A_{m}^{-\top} P+P A_{m}^{-1}\right)=-\frac{1}{2} A_{m}^{-\top} Q A_{m}^{-1}<0 \\
N=\frac{1}{2}\left(P A_{m}^{-1}-A_{m}^{-\top} P\right) .
\end{gathered}
$$

Since the symmetric part $M<0$, therefore $P A_{m}^{-1}<0$. Thus, $\dot{V}(e, \tilde{\Theta})$ becomes

$$
\dot{V}(e, \tilde{\Theta})=-e^{\top} Q e-2 e^{\top} P w-v x^{\top} \tilde{\Theta} B^{\top} A_{m}^{-\top} Q A_{m}^{-1} B \tilde{\Theta}^{\top} x+2 v x^{\top} \Theta^{*} B^{\top} P A_{m}^{-1} B \tilde{\Theta}^{\top} x
$$

and is bounded by

$$
\dot{V}(e, \tilde{\Theta}) \leq-\lambda_{\min }(Q)\|e\|^{2}+2 \lambda_{\max }(P)\|e\| w_{0}-v \lambda_{\min }\left(B^{\top} A_{m}^{-\top} Q A_{m}^{-1} B\right)\|x\|^{2}\|\tilde{\Theta}\|^{2}+2 v\left\|B^{\top} P A_{m}^{-1} B\right\|\|x\|^{2}\|\tilde{\Theta}\| \Theta_{0}
$$

Let $c_{1}=\lambda_{\min }(Q)>0, c_{2}=\frac{\lambda_{\max }(P) w_{0}}{c_{1}}>0, c_{3}=\lambda_{\min }\left(B^{\top} A_{m}^{-\top} Q A_{m}^{-1} B\right)>0$, and $c_{4}=\frac{\left\|B^{\top} P A_{m}^{-1} B\right\| \Theta_{0}}{c_{3}}>0$. Then, upon completing the squares, one gets

$$
\dot{V}(e, \tilde{\Theta}) \leq-c_{1}\left(\|e\|-c_{2}\right)^{2}+c_{1} c_{2}^{2}-v c_{3}\|x\|^{2}\left(\|\tilde{\Theta}\|-c_{4}\right)^{2}+v c_{3} c_{4}^{2}\|x\|^{2}
$$

$\dot{V}(e, \tilde{\Theta}) \leq 0$ implies

$$
\begin{aligned}
& \|e\| \geq c_{2}+\sqrt{c_{2}^{2}+\frac{v c_{3} c_{4}^{2}\|x\|^{2}}{c_{1}}}=r \\
& \|\tilde{\Theta}\| \geq c_{4}+\sqrt{c_{4}^{2}+\frac{c_{1} c_{2}^{2}}{v c_{3}\|x\|^{2}}}=\alpha
\end{aligned}
$$

Note that the lower bounds $r$ and $\alpha$ are dependent on $\|x\|$. Therefore, to prove boundedness, we also need to show that $\|x\|$ is bounded. To this end, we see that Eq. (19)

$$
-c_{1}\|e\|^{2}+2 c_{1} c_{2}\|e\|+v c_{3} c_{4}^{2}\|x\|^{2} \leq 0
$$

is equivalent to

$$
-e^{\top} Q e-2 e^{\top} P w+v c_{3} c_{4}^{2}\|x\|^{2} \leq 0
$$

There are two cases to consider:

1. The closed-loop plant with the nominal controller and no disturbance is stable. This is the same as stating that the uncertainty is a non-destabilizing uncertainty. Then, the error equation of the open-loop system is

$$
\dot{e}=A_{m} e-B \Theta^{* \top} x
$$

Choose a Lyapunov candidate function

$$
V(e)=e^{\top} P e
$$

Then

$$
\dot{V}(e)=-e^{\top} Q e-2 e^{\top} P B \Theta^{* \top} x \leq-c_{1}\|e\|^{2}+2\|e\|\|P B\| \Theta_{0} x
$$

Since the plant is stable, $\dot{V}(e) \leq 0$ which implies $\|x\|$ is bounded by

$$
\|x\| \leq \frac{c_{1}\|e\|}{2\|P B\| \Theta_{0}}
$$

Substituting into Eq. (22) yields

$$
-c_{1}\|e\|^{2}+2 c_{1} c_{2}\|e\|+\frac{v c_{3} c_{4}^{2} c_{1}^{2}\|e\|^{2}}{4\|P B\|^{2} \Theta_{0}^{2}} \leq 0
$$


For stability, the coefficient of $\|e\|^{2}$ must be negative. Therefore, there exists a maximum value $v_{\max }$ for which $0<v<v_{\max }$ where

$$
v_{\max }=\frac{4\|P B\|^{2} \Theta_{0}^{2}}{c_{1} c_{3} c_{4}^{2}}=\frac{4 \lambda_{\min }\left(B^{\top} A_{m}^{-\top} Q A_{m}^{-1} B\right)\|P B\|^{2}}{\lambda_{\min }(Q)\left\|B^{\top} P A_{m}^{-1} B\right\|^{2}}
$$

Note that $v_{\max }$ is independent of the bound of the parametric uncertainty $\Theta_{0}$ if the uncertainty is non-destabilizing. $\dot{V}(e, \tilde{\Theta})$ for the closed-loop system then becomes

$$
\dot{V}(e, \tilde{\Theta}) \leq-c_{1}\left(1-\frac{v}{v_{\max }}\right)\left(\|e\|-\frac{c_{2}}{1-\frac{v}{v_{\max }}}\right)^{2}+\frac{c_{1} c_{2}^{2}}{1-\frac{v}{v_{\max }}}-v c_{3}\|x\|^{2}\left(\|\tilde{\Theta}\|-c_{4}\right)^{2}
$$

Therefore, $\dot{V}(e, \tilde{\Theta}) \leq 0$ for the adaptive system implies either

$$
\|e\| \geq \frac{2 c_{2}}{1-\frac{v}{v_{\max }}}=r
$$

or

$$
\|\tilde{\Theta}\| \geq c_{4}+\sqrt{\frac{c_{1} c_{2}^{2}}{v c_{3}\|x\|^{2}\left(1-\frac{v}{v_{\max }}\right)}}=c_{4}+\sqrt{\frac{4\|P B\|^{2} \Theta_{0}^{2}\left(1-\frac{v}{v_{\max }}\right)}{v c_{1} c_{3}}}=\alpha
$$

The closed-loop system is uniformly ultimately bounded with

$$
\begin{gathered}
r \leq\|e\| \leq \rho=\sqrt{\frac{\lambda_{\max }(P) r^{2}+\lambda_{\max }\left(\Gamma^{-1}\right) \alpha^{2}}{\lambda_{\min }(P)}} \\
\alpha \leq\|\tilde{\Theta}\| \leq \beta=\sqrt{\frac{\lambda_{\max }(P) r^{2}+\lambda_{\max }\left(\Gamma^{-1}\right) \alpha^{2}}{\lambda_{\min }\left(\Gamma^{-1}\right)}}
\end{gathered}
$$

2. The closed-loop plant with the nominal controller has no stability guarantee. The uncertainty can be destabilizing. Since $e=x_{m}-x$, Eq. (22) can be expressed as

$$
-\left(x_{m}-x\right)^{\top} Q\left(x_{m}-x\right)-2\left(x_{m}-x\right)^{\top} P w+v c_{3} c_{4}^{2}\|x\|^{2} \leq 0
$$

Therefore

$$
-c_{1}\left\|x_{m}\right\|^{2}+2 c_{5}\left\|x_{m}\right\|\|x\|-c_{1}\|x\|^{2}+2 c_{1} c_{2}\left\|x_{m}\right\|+2 c_{1} c_{2}\|x\|+v c_{3} c_{4}^{2}\|x\|^{2} \leq 0
$$

where $c_{5}=\lambda_{\max }(Q)>c_{1}>0$.

The adaptive system is stable if $c_{1}-v c_{3} c_{4}^{2}>0$ which yields

$$
v_{\max }=\frac{c_{1}}{c_{3} c_{4}^{2}}=\frac{\lambda_{\min }(Q) \lambda_{\min }\left(B^{\top} A_{m}^{-\top} Q A_{m}^{-1} B\right)}{\left\|B^{\top} P A_{m}^{-1} B\right\|^{2} \Theta_{0}^{2}}
$$

Note that $v_{\max }$ is now dependent on the upper bound of the parametric uncertainty $\Theta_{0}$. As the bound of the uncertainty increases, $v_{\max }$ must be reduced to ensure stability of the closed-loop system. Thus, stability of the optimal control modification depends on the characteristics of the uncertainty. The adaptive law is guaranteed to be stable if a priori knowledge of the bound of the uncertainty exists.

$\|x\|$ is then bounded from below by

$$
\|x\| \geq \frac{c_{2}+c_{5}\left\|x_{m}\right\|_{\infty}+\sqrt{\left(c_{1} c_{2}+c_{5}\left\|x_{m}\right\|_{\infty}\right)^{2}+4\left(c_{1}+v c_{3} c_{4}^{2}\right)\left(2 c_{1} c_{2}\left\|x_{m}\right\|_{\infty}-c_{1}\left\|x_{m}\right\|_{\infty}^{2}\right)}}{c_{1}-v c_{3} c_{4}^{2}}
$$


The modification parameter $v$ for stability guarantee is dependent on the a priori knowledge of the bounds on the uncertainty as well as the disturbance to guarantee stability. Moreover, in the presence of a disturbance, i.e., $c_{2} \neq 0$, then for the standard MRAC which corresponds to $v=0$, the lower bound of $\tilde{\Theta}(t)$ is unbounded, as seen from Eq. (20). This implies an unbounded parameter variation for MRAC in the presence of a disturbance. This observation is consistent with the parameter drift phenomenon that the standard MRAC exhibits when a persistent excitation is not present in an adaptive regulator design.

Example 1: Consider a first-order SISO system

$$
\dot{x}=a x+b u+w
$$

where $a$ is unknown, $b$ is known, and $w(t)$ is a bounded disturbance where

$$
w=p(1+t)^{p-1}-a(1+t)^{p}+\gamma b^{2} \frac{(1+t)^{2 p+1}-1}{2 p+1}(1+t)^{p}
$$

An adaptive regulator is designed with the standard MRAC as

$$
\begin{gathered}
u=\bar{k}_{x} x \\
\dot{\bar{k}}_{x}=-\gamma x^{2} b
\end{gathered}
$$

with $\bar{k}_{x}(0)=0$.

The behaviors of the closed-loop system with the standard MRAC are shown in the following table.

\begin{tabular}{|c|c|c|c|c|}
\hline$p$ & $x(t)$ & $u(t)$ & $w(t)$ & $k_{x}(t)$ \\
\hline \hline$p>0$ & $\notin \mathscr{L}_{\infty}$ & $\notin \mathscr{L}_{\infty}$ & $\notin \mathscr{L}_{\infty}$ & $\notin \mathscr{L}_{\infty}$ \\
\hline$-\frac{1}{3}<p \leq 0$ & $\in \mathscr{L}_{\infty}$ & $\notin \mathscr{L}_{\infty}$ & $\notin \mathscr{L}_{\infty}$ & $\notin \mathscr{L}_{\infty}$ \\
\hline$-\frac{1}{2}<p \leq-\frac{1}{3}$ & $\in \mathscr{L}_{\infty}$ & $\in \mathscr{L}_{\infty}$ & $\in \mathscr{L}_{\infty}$ & $\notin \mathscr{L}_{\infty}$ \\
\hline$p=-\frac{1}{2}$ & $\in \mathscr{L}_{\infty}$ & $\notin \mathscr{L}_{\infty}$ & $\notin \mathscr{L}_{\infty}$ & $\notin \mathscr{L}_{\infty}$ \\
\hline$p<-\frac{1}{2}$ & $\in \mathscr{L}_{\infty}$ & $\in \mathscr{L}_{\infty}$ & $\in \mathscr{L}_{\infty}$ & $\in \mathscr{L}_{\infty}$ \\
\hline
\end{tabular}

Table 1 - Boundedness of Example Adaptive System

For $-\frac{1}{2}<p \leq-\frac{1}{3}, \bar{k}_{x}(t)$ becomes unbounded as $t \rightarrow \infty$, whereas $x(t), u(t)$, and $w(t)$ remain bounded. This demonstrates the parameter drift phenomenon of the standard MRAC. If $p<-\frac{1}{2}$, then all signals are bounded.

The adaptive regulator is redesigned with the optimal control modification adaptive law

$$
\dot{k}_{x}=-\gamma\left(x^{2} b-v x^{2} b^{2} a_{m}^{-1} k_{x}\right)
$$

where $a_{m}<0$ is chosen such that $a_{m}=a+b k_{x}^{*}$.

Let $w_{0}=\max |w|$ and $k_{0}=\left|k_{x}^{*}\right|$. Choose $Q=1, P=-\frac{a_{m}^{-1}}{2}$. Then $c_{1}=1, c_{2}=-\frac{a_{m}^{-1} w_{0}}{2}, c_{3}=b^{2} a_{m}^{-2}$, and $c_{4}=\frac{k_{0}}{2}$. The ultimate bound of $|x|$ is expressed as

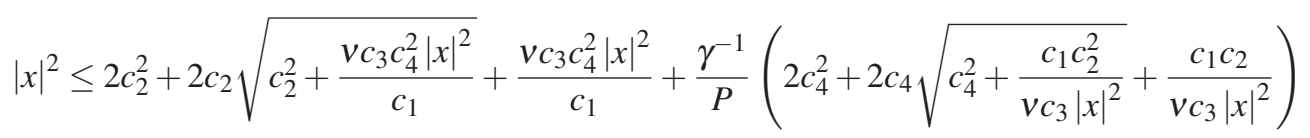

$v_{\max }$ is determined from the inequality in the limit as $|x| \rightarrow \infty$. This yields $v_{\max }=\frac{4}{b^{2} a_{m}^{-2} k_{0}^{2}}$. The ultimate bound of $|x|$ is the largest solution of the inequality for any $0<v<v_{\max }$.

The adaptive parameter $k_{x}(t)$ with the optimal control modification can be expressed in terms of the adaptive parameter $\bar{k}_{x}(t)$ with the standard MRAC as

$$
\frac{\dot{k}_{x}}{1-v a_{m}^{-1} b k_{x}}=\dot{\bar{k}}_{x}
$$

The solution of $k_{x}(t)$ has a closed form which is given by

$$
k_{x}=\frac{1}{v a_{m}^{-1} b}-\frac{1-v a_{m}^{-1} b k_{x}(0)}{v a_{m}^{-1} b} \exp \left\{-v a_{m}^{-1} b\left[\bar{k}_{x}-\bar{k}_{x}(0)\right]\right\}
$$


The standard MRAC exhibits a parameter drift as $\bar{k}_{x}(t) \rightarrow-\infty$. With the optimal control modification, $k_{x}(t)$ is bounded as $\vec{k}_{x}(t) \rightarrow-\infty$. Therefore, the solution is guaranteed to be stable in the presence of a disturbance for $0<v<\frac{4}{b^{2} a_{m}^{-2} k_{0}^{2}}$.

The responses of the closed-loop system with $a=1, b=1, p=-\frac{5}{12}, \gamma_{x}=10$, and $x(0)=1$ with the standard MRAC and the optimal control modification with $v=0.1$ are shown in Figs. 1 and 2, respectively.
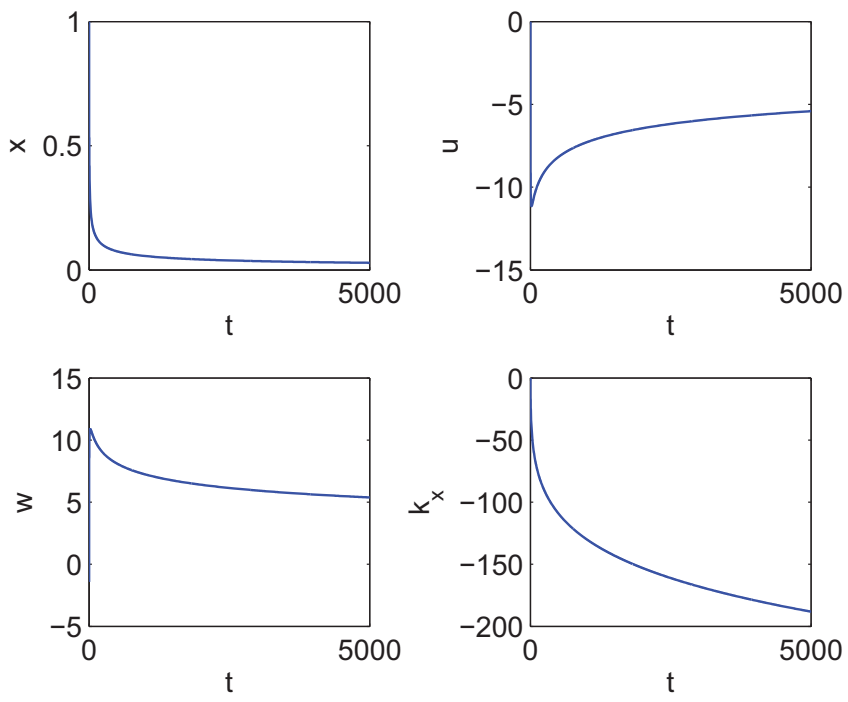

Fig. 1 - Parameter Drift of MRAC for $p=-\frac{5}{12}$ and $\gamma_{x}=10$
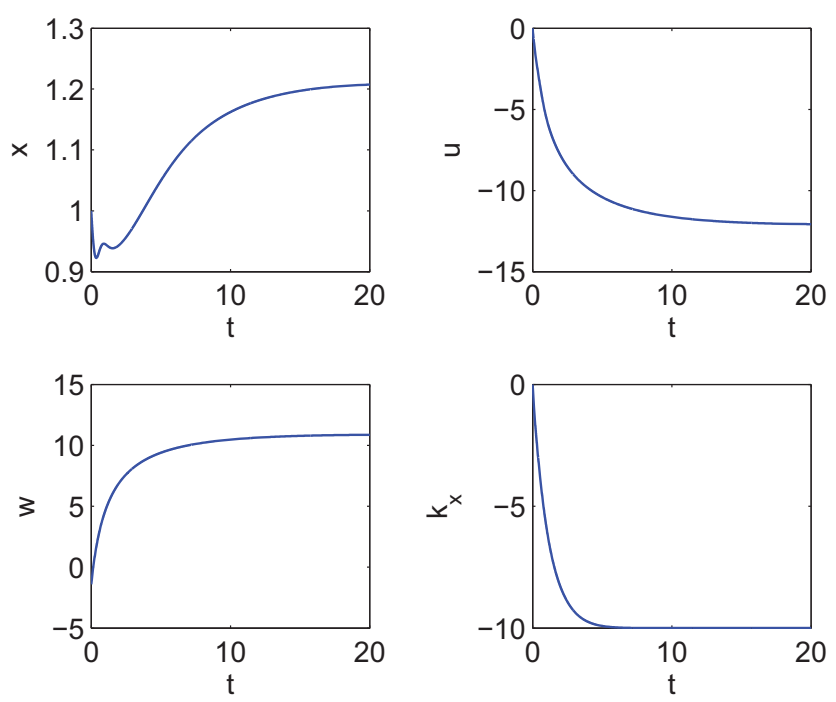

Fig. 2 - Optimal Control Modification with $v=0.1$ for $p=-\frac{5}{12}$ and $\gamma_{x}=10$

\section{Linear Asymptotic Property}

It has been shown that MRAC is non-robust with fast adaptation. The time delay margin of MRAC tends to zero as the adaptive gain tends to infinity. The optimal control modification adaptive law exhibits a linear asymptotic property as $\Gamma \rightarrow \infty$ or $t \rightarrow \infty$. If the closed-loop system is stable, then $\dot{\Theta} \rightarrow 0$ which implies

$$
\Theta^{\top} x \rightarrow \frac{1}{v}\left(B^{\top} A_{m}^{-\top} P B\right)^{-1} B^{\top} P e
$$


The closed-loop system then tends to an asymptotic linear system

$$
\dot{x}=\left(A_{m}+\frac{1}{v} P^{-1} A_{m}^{\top} P+B \Theta^{*^{\top}}\right) x-\frac{1}{v} P^{-1} A_{m}^{\top} P x_{m}+B_{m} r
$$

This system is stable for all $v>0$ if $A_{m}+B \Theta^{*^{\top}}$ is Hurwitz, but stable for $0<v<v_{\max }$ if $A_{m}+B \Theta^{*^{\top}}$ is not Hurwitz.

Consider a special case when $v=1$ corresponding to the optimal solution of the adaptive law, then

$$
A_{m}+P^{-1} A_{m}^{\top} P=P^{-1}\left(P A_{m}+A_{m}^{\top} P\right)=-P^{-1} Q
$$

Since $P>0$ and $Q>0,-P^{-1} Q<0$. The closed-loop poles of the ideal system with $\Theta^{*}=0$ are all negative real. The ideal system is exponentially stable with no high-frequency oscillations.

The linear asymptotic property of the optimal control modification is quite useful since it can be analyzed using many existing linear analysis tools. Moreover, because of its linear asymptotic property, the closed-loop system has a scaled input-out behavior as $\Gamma \rightarrow \infty$. That is, if $r(t)$ is scaled by a multiplier $c$, then $x(t)$ is scaled by the same amount.

Theorem 2: Let $x(t)=x_{0}(t)$ be the response due to $r(t)=r_{0}(t)$, then if $r(t)=c r_{0}(t)$ where $c$ is a constant, then it follows that $x(t)=c x_{0}(t)$ as $\Gamma \rightarrow \infty$.

Proof: Since the the asymptotic closed-loop system is linear time invariant, then

$$
x=\left(s I-A_{m}-\frac{1}{v} P^{-1} A_{m}^{\top} P-B \Theta^{*^{\top}}\right)^{-1}\left(-\frac{1}{v} P^{-1} A_{m}^{\top} P x_{m}+B_{m} r\right)
$$

The transfer function of the reference model is

$$
\frac{x_{m}}{r}=\left(s I-A_{m}\right)^{-1} B_{m}
$$

Then the closed-loop transfer function is obtained as

$$
\frac{x}{r}=\left(s I-A_{m}-\frac{1}{v} P^{-1} A_{m}^{\top} P-B \Theta^{*^{\top}}\right)^{-1}\left[-\frac{1}{v} P^{-1} A_{m}^{\top} P\left(s I-A_{m}\right)^{-1}+I\right] B_{m}
$$

Thus, if $r(t)=c r_{0}(t)$, then $x(t)=c x_{0}(t)$. The scale input-output behavior makes the optimal control modification more predictable than the $\sigma$ modification and $e$ modification, both of which do not exhibit linear asymptotic properties for linear uncertain plants.

The equilibrium value of $x(t)$ as $t \rightarrow \infty$ can be found by setting $s=0$

$$
\bar{x}=-\left(A_{m}+\frac{1}{v} P^{-1} A_{m}^{\top} P+B \Theta^{*^{\top}}\right)^{-1}\left(\frac{1}{v} P^{-1} A_{m}^{\top} P A_{m}^{-1}+I\right) B_{m} r
$$

If $v=0$, then the ideal property of asymptotic tracking of MRAC is recovered since

$$
\begin{aligned}
\bar{x}=-\lim _{v \rightarrow 0}\left(A_{m}+\frac{1}{v} P^{-1} A_{m}^{\top} P+B \Theta^{*^{\top}}\right)^{-1}( & \left.\frac{1}{v} P^{-1} A_{m}^{\top} P A_{m}^{-1}+I\right) B_{m} r \\
& =-\lim _{v \rightarrow 0}\left(\frac{1}{v} P^{-1} A_{m}^{\top} P\right)^{-1} \frac{1}{v} P^{-1} A_{m}^{\top} P A_{m}^{-1} B_{m} r=-A_{m}^{-1} B_{m} r=\bar{x}_{m}
\end{aligned}
$$

The equilibrium value of the tracking error is given by

$$
\bar{e}=\bar{x}_{m}-\bar{x}=\left[-A_{m}^{-1}+\left(A_{m}+\frac{1}{v} P^{-1} A_{m}^{\top} P+B \Theta^{*^{\top}}\right)^{-1}\left(\frac{1}{v} P^{-1} A_{m}^{\top} P A_{m}^{-1}+I\right)\right] B_{m} r
$$

The largest norm of $\bar{e}(t)$ which can be interpreted as a steady state error when $\Gamma \rightarrow \infty$ is given by

$$
\|\bar{e}\|=\left\|-A_{m}^{-1} B_{m}+\left(A_{m}+\frac{1}{v} P^{-1} A_{m}^{\top} P+B \Theta^{*}\right)^{-1}\left(\frac{1}{v} P^{-1} A_{m}^{\top} P A_{m}^{-1}+I\right) B_{m}\right\|\|r\|_{\infty}
$$


The linear asymptotic property also affords another advantage in that the stability margins of the system can be computed.

Consider a first-order time-delay SISO system with an optimal control modification adaptive controller

$$
\begin{gathered}
\dot{x}=a x+b\left[u\left(t-t_{d}\right)+\theta^{*} x\right] \\
u=k_{x} x+k_{r} r-\theta(t) x \\
\dot{\theta}=-\gamma\left(x e b-v x^{2} a_{m}^{-1} b^{2} \theta\right)
\end{gathered}
$$

with $a$ and $b$ known and $a_{m}=a+b k_{x}<0$.

As $\gamma \rightarrow \infty$, the equilibrium value of $\theta x$ is

$$
\bar{\theta} x=\frac{x_{m}-x}{v a_{m}^{-1} b}
$$

Then the closed-loop plant is given by

$$
\dot{x}=\left(a+b \theta^{*}\right) x+\left(b k_{x}+\frac{a_{m}}{v}\right) x\left(t-t_{d}\right)-\frac{a_{m}}{v} x_{m}\left(t-t_{d}\right)+b k_{r} r\left(t-t_{d}\right)
$$

To simplify the analysis, let $r(t)=1$ and $x_{m}(t)=1$. Then the characteristic equation with $s=j \omega$ is

$$
j \omega-\left(a+b \theta^{*}\right)-\left(b k_{x}+\frac{a_{m}}{v}\right)\left(\cos \omega t_{d}-j \sin \omega t_{d}\right)=0
$$

which results in the following equations

$$
\begin{gathered}
-\left(a+b \theta^{*}\right)-\left(b k_{x}+\frac{a_{m}}{v}\right) \cos \omega t_{d}=0 \\
\omega+\left(b k_{x}+\frac{a_{m}}{v}\right) \sin \omega t_{d}=0
\end{gathered}
$$

The crossover frequency and time delay margin are computed as

$$
\begin{gathered}
\omega=\sqrt{\left(b k_{x}+\frac{a_{m}}{v}\right)^{2}-\left(a+b \theta^{*}\right)^{2}} \\
t_{d}=\frac{1}{\omega} \cos ^{-1}\left(-\frac{a+b \theta^{*}}{b k_{x}+\frac{a_{m}}{v}}\right)
\end{gathered}
$$

If $v=0$, then the optimal control modification reverts to the standard MRAC whereupon

$$
\begin{gathered}
\omega=\lim _{v \rightarrow 0} \sqrt{\left(b k_{x}+\frac{a_{m}}{v}\right)^{2}-\left(a+b \theta^{*}\right)^{2}} \rightarrow \infty \\
t_{d}=\lim _{v \rightarrow 0} \frac{1}{\omega} \cos ^{-1}\left(-\frac{a+b \theta^{*}}{b k_{x}+\frac{a_{m}}{v}}\right)=0
\end{gathered}
$$

Thus the time delay margin for the standard MRAC goes to zero as the adaptive gain goes to infinity. On the other hand, for any $0<v<v_{\max }$, the optimal control modification adaptive law yields a non-zero time delay margin as the adaptive gain goes to infinity. This robustness property is required to maintain a sufficient stability margin of the closed-loop system. For a given time delay margin $t_{d}$ and specification of $\theta^{*}$, the modification parameter $v$ thus can be computed to guarantee stability of the closed-loop system.

Example 2: Let $a=a_{m}=-1, b=b_{m}=1, \theta^{*}=2$, and $r(t)=1$. The open-loop system is unstable. So the limiting value of $v$ can be computed from Theorem 1 and from the linear asymptotic property as

$$
v_{\text {max }}=\min \left[\left(\frac{2 a_{m}}{b \theta^{*}}\right)^{2},-\frac{a_{m}}{a_{m}+b \theta^{*}}\right]=\min (1,1)=1
$$

Choose $v=0.2<1$. The time delay margin for the closed-loop system with the optimal control modification is calculated as

$$
\omega=\sqrt{\frac{1}{v^{2}}-1}=4.8990 \mathrm{rad} / \mathrm{sec}
$$




$$
t_{d}=\frac{1}{\sqrt{\frac{1}{v^{2}}-1}} \cos ^{-1} v=0.2795 \mathrm{sec}
$$

Since $t_{d}$ decreases with $\gamma$, the time delay margin estimated with $\gamma \rightarrow \infty$ is the minimum value of the time delay margin for any finite $\gamma<\infty$. We see that for a small value of $v$, a significant increase in the time delay margin can be achieved.

The steady state error is estimated to be

$$
\bar{e}=\left[-a_{m}^{-1}+\left(a_{m}+\frac{a_{m}}{v}+b \theta^{*}\right)^{-1}\left(\frac{1}{v}+1\right)\right] b_{m} r=-0.5
$$

The equilibrium value of $\theta$ is computed to be

$$
\bar{\theta}=\frac{\bar{e}}{v a_{m}^{-1} b \bar{x}}=\frac{\bar{e}}{v a_{m}^{-1} b\left(\bar{x}_{m}-\bar{e}\right)}=1.6667
$$

A small time delay of $0.0020 \mathrm{sec}$ in injected at the input in the simulation. This time delay causes the closed-loop system with the standard MRAC to go unstable. On the other hand, The closed-loop system with the optimal control modification is completely stable. The response of the closed-loop system with the optimal control modification is shown in Fig. 3. The simulation results agree very well with the equilibrium values of $\bar{e}$ and $\bar{\theta}$.
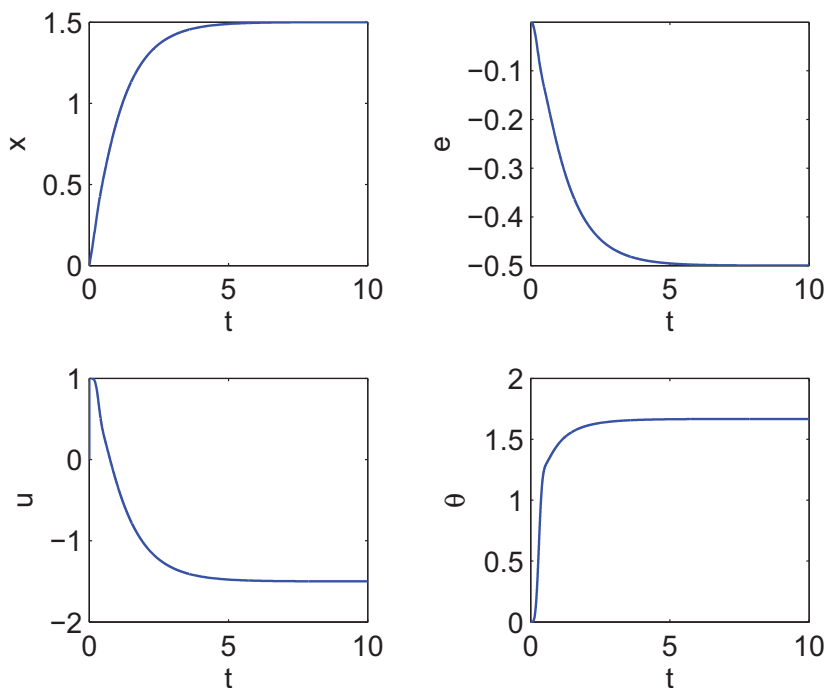

Fig. 3 - Optimal Control Modification with Fast Adaptation

When a time delay equal to the time delay margin of $0.2795 \mathrm{sec}$ is injected at the input, the closed-loop system begins to diverge, as shown in Fig. 4. Thus, the numerical evidence of the time delay margin is also in agreement with the analytical result. It should be noted that the time delay margin cannot be computed analytically with the $\sigma$ modification and $e$ modification because they do not possess linear asymptotic properties.

To illustrate the scaled input-output linear behavior of the optimal control modification adaptive law, the reference command signal is doubled so that $r(t)=2$. The simulation results are shown in Fig. 5. Both the reference model and the closed-loop system with the optimal control modification exhibit the scaled input-output property. For the optimal control modification, $\bar{x}=3$ for $r(t)=2$ which is twice $\bar{x}=1.5$ for $r(t)=1$.

For comparison, the closed-loop responses with both the $\sigma$ modification and $e$ modification are computed. For the $\sigma$ modification with $\sigma=0.2, \bar{x}=2.1763$ for $r(t)=2$ versus $\bar{x}=1.2787$ for $r(t)=1$. For the $e$ modification with $\mu=0.2, \bar{x}=1.9725$ for $r(t)=2$ versus $\bar{x}=1.0015$ for $r(t)=1$. Moreover, the $e$ modification also exhibits oscillations with different frequencies and amplitudes as the reference command signal is doubled. This example illustrates that even if the open-loop uncertain system is linear, the closed-loop behaviors with the $\sigma$ modification and $e$ modification are completely nonlinear. The closed-loop system with the optimal control modification adaptive law, on the other hand, tends to a linear system as the adaptive gain tends to infinity. 

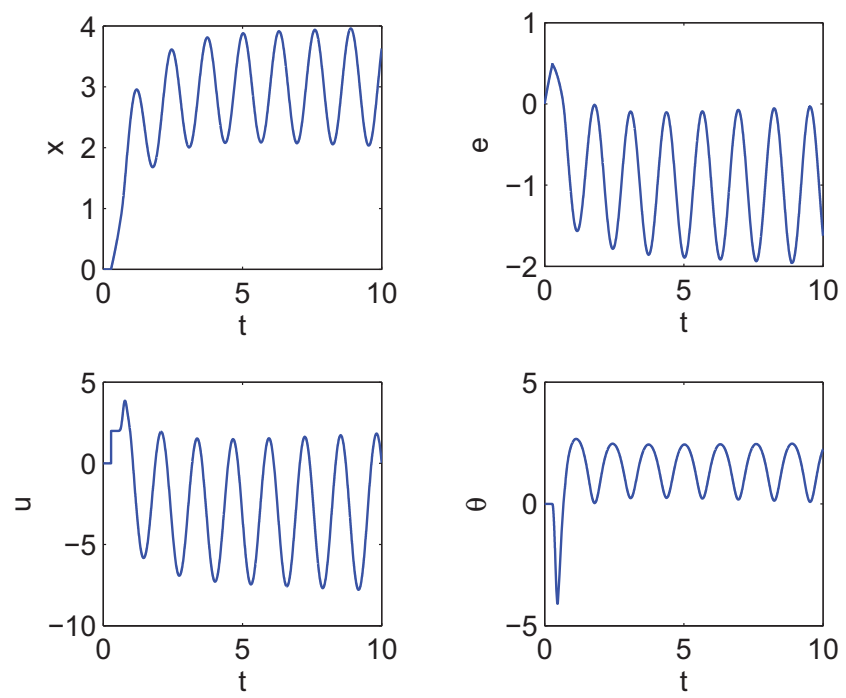

Fig. 4 - Optimal Control Modification with $t_{d}=0.2795 \mathrm{sec}$
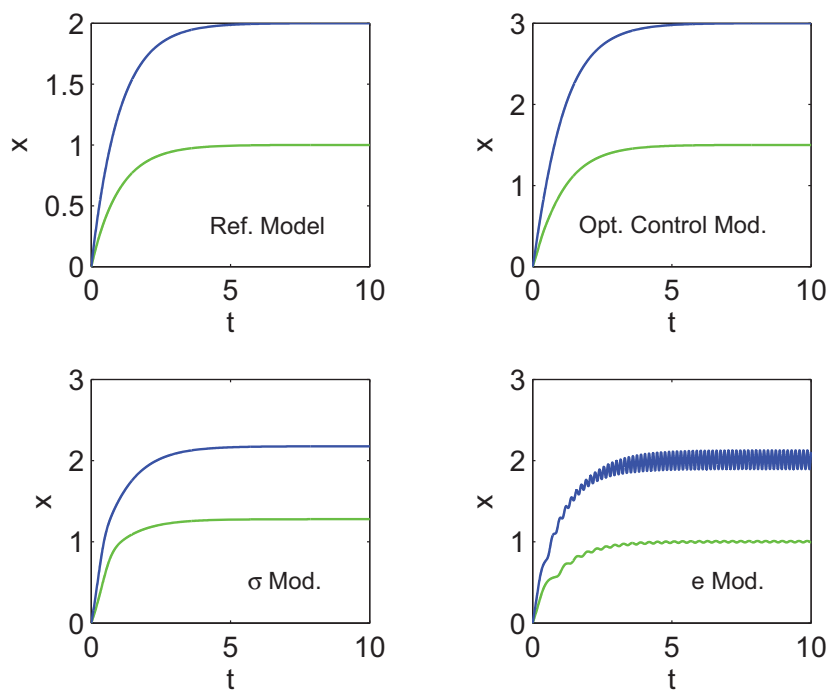

Fig. 5 - Scaled Input-Output Property of Adaptive Control

\section{Linear Systems with Unmodeled Dynamics and Rohrs Counterexample}

Consider the system

$$
\begin{gathered}
\dot{x}=A x+B u+\Delta(x, z, u) \\
\dot{z}=f(x, z, u) \\
y=x
\end{gathered}
$$

where $z$ is the unmeasurable and unobservable state vector, $\Delta$ is the plant model error that is unknown and not accounted for, $\dot{z}$ is the unmodeled dynamics, and $y$ is the plant output vector which is just the measurable state vector $x$.

If model-reference adaptive control is used in a control design by assuming $\Delta=0$, then it is clear that such a control design can be non-robust as demonstrated by instability phenomena of MRAC for non-minimum phase systems.

Research in robust adaptive control was motivated by instability phenomena of adaptive control. In fact, instability of adaptive control in the early 1960's which contributed to the crash of one of the NASA X-15 hypersonic vehicles 
caused a great deal of concern about the viability of adaptive control. Rohrs et al. investigated various instability mechanisms of adaptive control due to unmodeled dynamics in a paper entitled "Robustness of Adaptive Control in the Presence of Unmodeled Dynamics" published in 1982. ${ }^{1}$ The Rohrs counterexample demonstrates the weakness of MRAC in its lack of robustness.

The Rohrs counterexample is described by a first-order system with a pair of complex unmodeled poles

$$
y=\frac{2}{s+1} \frac{229}{s^{2}+30 s+229} u
$$

The system has a relative degree 3 and is minimum phase.

The reference model is specified as

$$
y_{m}=\frac{3}{s+3} r
$$

The reference model is strictly positive real (SPR) with relative degree 1 . Since the relative degree of the reference model is less than the relative degree of the plant, perfect tracking is not possible. Adaptive control of systems with relative degrees greater than 1 is generally more difficult since the model reference cannot be chosen to be SPR. ${ }^{9}$

The controller is given by

$$
\begin{gathered}
u=k_{y}(t) y+k_{r}(t) r \\
\dot{k}_{y}=\gamma_{x} y e \\
\dot{k}_{r}=\gamma_{r} r e
\end{gathered}
$$

where $e=y_{m}-y$.

$k_{y}$ and $k_{r}$ are initialized with $k_{y}(0)=-0.65$ and $k_{r}(0)=1.14$.

The reference command signal is given by

$$
r=0.3+1.85 \sin 16.1 t
$$

where the frequency $16.1 \mathrm{rad} / \mathrm{sec}$ is the frequency at which the closed-loop transfer function with $k_{y}=k_{y}(0)$ has a $-180^{\circ}$ phase shift which implies a $0^{\circ}$ phase margin. The closed-loop system is unstable, as shown in Fig. 6.
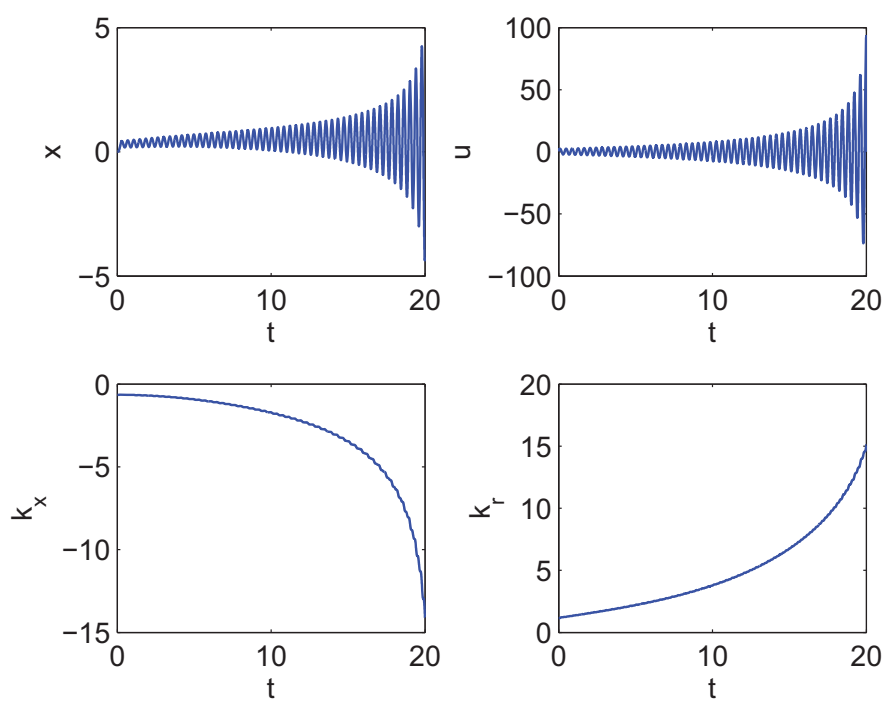

Fig. 6 - Instability of MRAC by Rohrs Counterexample

The Rohrs counterexample shows that the instability mechanisms can be due to the initialization of the adaptive parameters as well as the reference command signal. The underlying cause of instability is the lack of robustness of the closed-loop system. Changing either the initial condition of $k_{y}$ and or the frequency in the reference command signal can result in stabilization of the closed-loop system if it has a sufficient phase margin.

The optimal control modification adaptive law can be designed to handle linear systems with unmodeled dynamics by utilizing the linear asymptotic property. 
Suppose a plant with unmodeled dynamics is given by the following transfer function:

$$
\frac{y}{u}=W_{p}(s)=k_{p} \frac{Z_{p}(s)}{R_{p}(s)}
$$

where $k_{p}$ is a high-frequency gain, and $Z_{p}(s)$ and $R_{p}(s)$ are monic Hurwitz polynomials of degrees $m_{p}$ and $n_{p}$, respectively, and $n_{p}-m_{p}>1$ is the relative degree of the plant.

The reference model is given by a transfer function

$$
\frac{y_{m}}{r}=W_{m}(s)=k_{m} \frac{Z_{m}(s)}{R_{m}(s)}
$$

where $k_{m}$ is a high-frequency gain, and $Z_{m}(s)$ and $R_{m}(s)$ are monic Hurwitz polynomials of degrees $m_{m}$ and $n_{m}$, respectively, and $n_{m}-m_{m} \geq 1$ is the relative degree of the reference model.

Suppose the adaptive controller is designed with the optimal control modification as

$$
u=k_{y} y+k_{r} r
$$

where

$$
\begin{aligned}
& \dot{k}_{y}=\gamma_{y}\left(y e-v y^{2} k_{y}\right) \\
& \dot{k}_{r}=\gamma_{r}\left(r e-v r^{2} k_{r}\right)
\end{aligned}
$$

Let $n_{p}-m_{p}>n_{m}-m_{m}$. So the SPR condition is no longer possible to ensure tracking of the reference model. Stability of the closed-loop system cannot also be guaranteed with the standard MRAC.

Using the linear asymptotic property of the optimal control modification, the asymptotic value of the adaptive controller $u$ can be computed as $\gamma_{y} \rightarrow \infty$ and $\gamma_{r} \rightarrow \infty$.

$$
\bar{u}=\frac{2 y_{m}-2 y}{v}
$$

The asymptotic closed-loop transfer function can now be computed as

$$
\frac{\bar{y}}{r}=\frac{2 W_{p}(s) W_{m}(s)}{v+2 W_{p}(s)}=\frac{2 k_{m} k_{p} Z_{p}(s) Z_{m}(s)}{R_{m}(s)\left(v R_{p}(s)+2 k_{p} Z_{p}(s)\right)}
$$

By a suitable selection of the modification parameter $v$, the asymptotic closed-loop transfer function can be designed to have closed-loop stability. Once the modification parameter $v$ is chosen, the adaptive gains $\gamma_{y}$ and $\gamma_{r}$ can be selected to be any reasonable values without compromising closed-loop stability of the adaptive laws.

Referring back to the Rohrs counterexample, the adaptive controller asymptotically tends to

$$
\bar{u}=\frac{2 y_{m}-2 y}{v}=\frac{6 r}{v(s+3)}-\frac{2 y}{v}
$$

as $\gamma_{y} \rightarrow \infty$ and $\gamma_{r} \rightarrow \infty$.

Then the asymptotic closed-loop transfer function is obtained as

$$
\frac{\bar{y}}{r}=\frac{2748}{v(s+3)\left(s^{3}+31 s^{2}+259 s+229+\frac{916}{v}\right)}
$$

Note that the closed-loop transfer function has a relative degree 4 while the transfer function of the reference model has a relative degree 1 . This prevents the output $y$ from tracking $y_{m}$.

The characteristic equation of $\bar{y}$ with input time delay is

$$
s^{3}+31 s^{2}+259 s+229+\frac{916}{v} e^{-t_{d} s}=0
$$

Substituting $s=j \omega$ yields

$$
-j \omega^{3}-31 \omega^{2}+259 j \omega+229+\frac{916}{v}\left(\cos \omega t_{d}-j \sin \omega t_{d}\right)=0
$$


This results in two frequency equations

$$
\begin{aligned}
& -31 \omega^{2}+229+\frac{916}{v} \cos \omega t_{d}=0 \\
& -\omega^{3}+259 \omega-\frac{916}{v} \sin \omega t_{d}=0
\end{aligned}
$$

We then obtain the cross-over frequency and phase margin as functions of the modification parameter $v$

$$
\begin{gathered}
\omega^{6}+443 \omega^{4}+52883 \omega^{2}+229^{2}-\frac{916^{2}}{v^{2}}=0 \\
\phi=\omega t_{d}=\tan ^{-1}\left(\frac{-\omega^{3}+259 \omega}{31 \omega^{2}-229}\right)
\end{gathered}
$$

The plot of $v$ versus $\phi$ and $\omega$ is shown in Fig. 7. Notice that at $v=0.117, \omega=16.1 \mathrm{rad} / \mathrm{sec}$ and $\phi=0$. Note that the zero phase margin at the reference frequency of $16.1 \mathrm{rad} / \mathrm{sec}$ is consistent with the instability of the plant in the Rohrs counterexample. So the minimum value of $v$ is $v_{\text {min }}=0.117$. At $v=1$, the phase margin is about $78^{\circ}$.
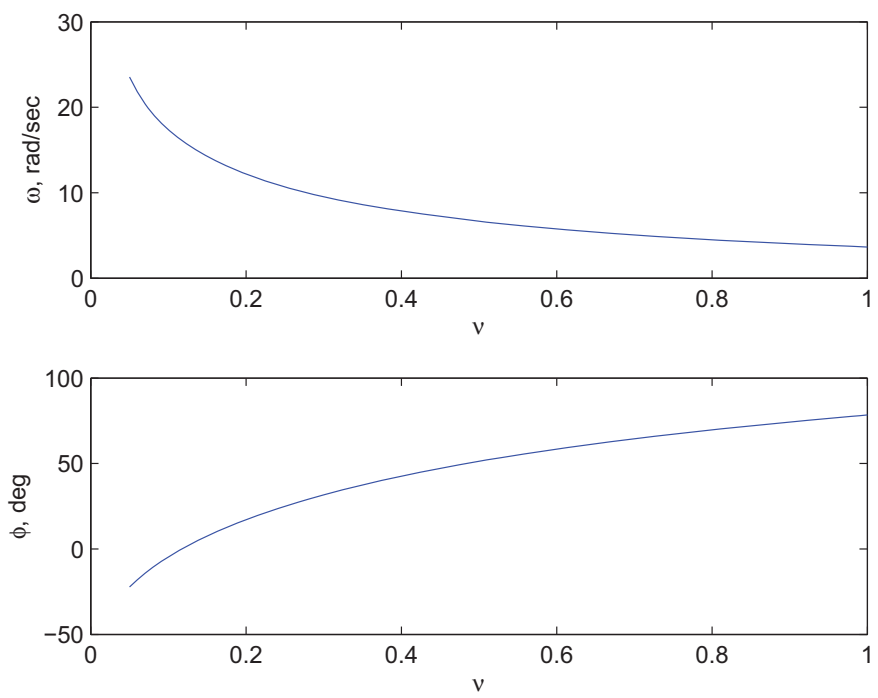

Fig. 7 - Phase Margin and Cross-Over Frequency of Rohrs Counterexample as Functions of $v$

Suppose a $45^{\circ}$ phase margin is select. Then, $\phi=45^{\circ}$ occurs at $v=0.426$ and $\omega=7.5099 \mathrm{rad} / \mathrm{sec}$. The asymptotic closed-loop transfer function with $v=0.426$ is

$$
\frac{\bar{y}}{r}=\frac{2748}{0.426 s^{4}+14.484 s^{3}+149.952 s^{2}+1344.556 s+3040.662}
$$

The closed-loop plant is robustly stable with a phase margin of $45^{\circ}$. The asymptotic response $\bar{y}(t)$ and control signal $\bar{u}(t)$ evaluated analytically agree very well with the simulation results of $y(t)$ and $r(t)$, as shown in Fig. 8 . Thus, the linear asymptotic property of the optimal control modification is demonstrated to be able to facilitate stability analysis of linear uncertain systems with unmodeled dynamics, time delay, or non-minimum phase behaviors.

All the three robust modification schemes; namely, $\sigma$ modification, $e$ modification , and optimal control modification exhibit minimum values of the modification parameters at which the plant in the Rohrs counterexample begins to stabilize. The $\sigma$ and $e$ modification parameters can be found by trial and error. In contrast, the modification parameter $v$ is found analytically by taking the advantage of the linear asymptotic property of the optimal control modification adaptive law. 

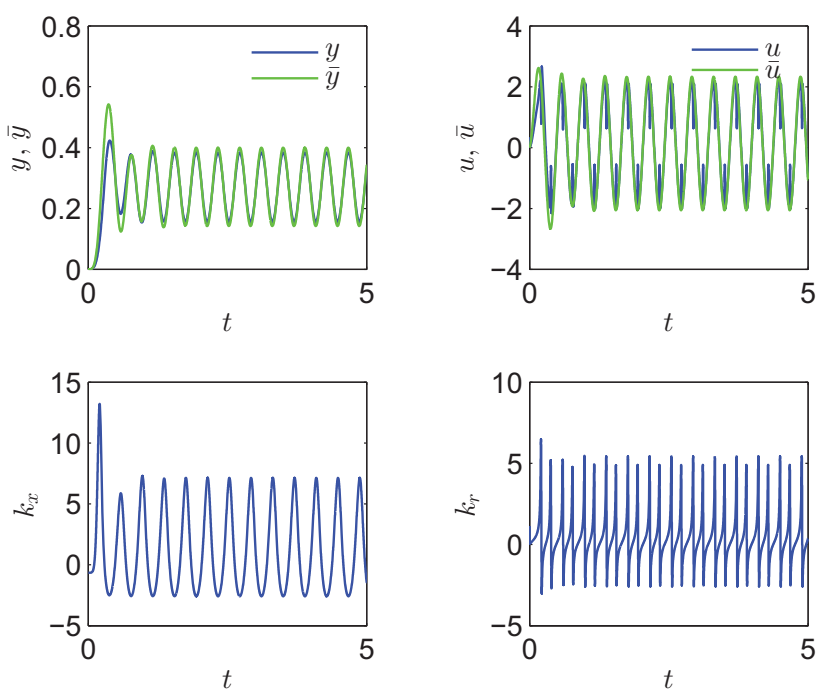

Fig. 8 - Closed-Loop Response of Rohrs Counterexample with Optimal Control Modification

\section{Adaptive Control of Non-Minimum Phase Plants with Relative Degree 1}

Non-minimum phase plants are well-known to be very difficult to control by adaptive control. Output feedback adaptive control generally relies on the SPR property to ensure stability. For non-minimum phase plants, the SPR property does not exist, thereby destroying the ability to analyze stability of adaptive control using the SPR property. The linear asymptotic property of the optimal control modification can be used to analyze non-minimum phase plants in some instances. We will demonstrate such an analysis with the following example as a motivation. In future work, we will further explore this property to address in general adaptive control of non-minimum phase plants.

Consider the following SISO plant

$$
\begin{gathered}
\dot{x}=a x+b u+g z \\
\dot{z}=h z+l x+m u \\
y=x
\end{gathered}
$$

where $z$ is the unmeasurable state with internal dynamics, and the parameters $a, g, h, l$, and $m$ are unknown, but $b$ is known.

The objective is to design an output feedback adaptive controller to enable tracking the following reference model

$$
y_{m}=W_{m}(s) r=k_{m} \frac{Z_{m}(s)}{R_{m}(s)} r=\frac{b_{m} r}{s-a_{m}}
$$

where $a_{m}<0$ and $k_{m}=b_{m}$.

The transfer functions of the plant is expressed as

$$
\frac{y}{u}=W_{p}(s)=k_{p} \frac{Z_{p}(s)}{R_{p}(s)}=\frac{b(s-h)+g m}{(s-a)(s-h)-g l}
$$

where $k_{p}=b$.

Note that $W_{m}$ is SPR with a relative degree 1. The plant is also with a relative degree 1 and is assumed to be stable, so $R_{p}(s)=(s-a)(s-h)-g l$ is Hurwitz.

The output feedback adaptive controller is desgined to be of the form

$$
u=\frac{b^{-1}\left(a_{m}-\theta_{1}\right) s y-b^{-1} \theta_{2} y+b^{-1} b_{m} s r}{s-b^{-1} \theta_{3}}
$$

where $\theta_{1}, \theta_{2}$, and $\theta_{3}$ are adaptive parameters. 
Then the closed-loop plant is written as

$$
y=\frac{\left(g m-b h-\theta_{3}\right) u+b_{m} s r}{s^{2}-\left(a_{m}+a+h-\theta_{1}\right) s-\left(g l-a h-\theta_{2}\right)}
$$

Let $\theta_{1}^{*}=a+h, \theta_{2}^{*}=g l-a h$, and $\theta_{3}^{*}=g m-b h$. Then, the tracking error equation is obtained as

$$
\dot{e}=a_{m} e+\tilde{\theta}_{1} y+\tilde{\theta}_{2} \int_{0}^{t} y d \tau+\tilde{\theta}_{3} \int_{0}^{t} u d \tau
$$

where $e=y_{m}-y, \tilde{\theta}_{1}=\theta_{1}-\theta_{1}^{*}, \tilde{\theta}_{2}=\theta_{2}-\theta_{2}^{*}$, and $\tilde{\theta}_{3}=\theta_{3}-\theta_{3}^{*}$.

The standard MRAC adaptive laws are

$$
\begin{gathered}
\dot{\theta}_{1}=-\gamma_{1} y e \\
\dot{\theta}_{2}=-\gamma_{2}\left(\int_{0}^{t} y d \tau\right) e \\
\dot{\theta}_{3}=-\gamma_{3}\left(\int_{0}^{t} u d \tau\right) e
\end{gathered}
$$

If $Z_{p}(s)$ is minimum phase, then $\theta_{1} \rightarrow \theta_{1}^{*}, \theta_{2} \rightarrow \theta_{2}^{*}$, and $\theta_{3} \rightarrow \theta_{3}^{*}$. The adaptive controller is stable and tends to the ideal controller

$$
u^{*}=\frac{b^{-1}\left[\left(a_{m}-\theta_{1}^{*}\right) s-\theta_{2}^{*}\right] y+b^{-1} b_{m} s r}{s+b^{-1} \theta_{3}^{*}}
$$

since $b^{-1} \theta_{3}^{*}>0$ if $Z_{p}(s)$ is minimum phase.

The stable zero of $Z_{p}(s)$ is cancelled by the stable pole of the adaptive controller to achieve perfect tracking of the reference model.

On the other hand, if $Z_{p}(s)$ is non-minimum phase, $b^{-1} \theta_{3}^{*}<0$. Then, the adaptive controller has an unstable pole. The standard MRAC adaptive law is therefore unstable.

Suppose the following optimal control modification adaptive laws are used:

$$
\begin{gathered}
\dot{\theta}_{1}=-\gamma_{1}\left(y e+v_{1} y^{2} \theta_{1}\right) \\
\dot{\theta}_{2}=-\gamma_{2}\left[\left(\int_{0}^{t} y d \tau\right) e+v_{2}\left(\int_{0}^{t} y d \tau\right)^{2} \theta_{2}\right] \\
\dot{\theta}_{3}=-\gamma_{3}\left[\left(\int_{0}^{t} u d \tau\right) e+v_{3}\left(\int_{0}^{t} u d \tau\right)^{2} \theta_{3}\right]
\end{gathered}
$$

where $v_{1}, v_{2}$, and $v_{3}$ are the modification parameter.

Then the linear asymptotic property yields the equilibrium values of $\theta_{1}, \theta_{2}$, and $\theta_{3}$

$$
\begin{aligned}
\theta_{1} y & =-\frac{y_{m}-y}{v_{1}} \\
\theta_{2} \frac{y}{s} & =\frac{y_{m}-y}{v_{2}} \\
\theta_{3} \frac{u}{s} & =\frac{y_{m}-y}{v_{3}}
\end{aligned}
$$

Then the adaptive controller tends to

$$
u=b^{-1} a_{m} y+b^{-1} \frac{y_{m}-y}{v_{1}}+b^{-1} \frac{y_{m}-y}{v_{2}}+b^{-1} \frac{y_{m}-y}{v_{3}}+b^{-1} b_{m} r
$$

Note that stability of the adaptive controller is no longer affected by the non-minimum phase of $Z_{p}(s)$. Stability of the closed-loop plant is then determined by a proper selection of the modification $v$.

Example 3: Consider the system

$$
\dot{x}=a x+u-2 z+w
$$




$$
\begin{gathered}
\dot{z}=-z+u \\
y=x
\end{gathered}
$$

where $a<0$ is unknown, but $a=-1$ for simulation purposes.

The system is non-minimum phase with a transfer function

$$
\begin{gathered}
y=\frac{(s-1) u+(s+1) w}{(s-a)(s+1)} \\
y=\frac{(s-1) u}{(s-a)(s+1)}
\end{gathered}
$$

An adaptive regulator is designed as

$$
\begin{gathered}
u=k_{y} y \\
\dot{k}_{y}=-\gamma_{y}\left(y^{2}+v y^{2} k_{y}\right)
\end{gathered}
$$

Since the system is non-minimum phase, the standard MRAC corresponding to $v=0$ will result in instability. Therefore, there exists a minimum value of $v$ that stabilizes the closed-loop system. Using the linear asymptotic property, we can identify this minimum value analytically.

Using the linear asymptotic property, we see that $\bar{k}_{y} \rightarrow-\frac{1}{v}$ as $\gamma_{y} \rightarrow \infty$. Therefore, the asymptotic closed-loop transfer function is

$$
\frac{\bar{y}}{w}=\frac{s+1}{(s-a)(s+1)+\frac{1}{v}(s-1)}
$$

The characteristic roots of the closed-loop transfer function are given by

$$
s=-\frac{1-a}{2}-\frac{1}{2 v} \pm \sqrt{\left(\frac{1-a}{2}+\frac{1}{2 v}\right)^{2}+a+\frac{1}{v}}
$$

Note that if $v<-\frac{1}{a}$, the closed-loop plant is unstable. So, $v \geq-\frac{1}{a}$ for closed-loop stability. The steady state closed-loop transfer function is equal to

$$
\frac{\bar{y}}{w}=-\frac{1}{a+\frac{1}{v}}
$$

Figure 9 shows the response of the closed-loop system with $v=2$ for a unit step disturbance $w(t)=1$. The numerical results are in agreement with the steady state value $\bar{k}_{y}(t)=-\frac{1}{2}$ and the steady state response $\bar{y}(t)=2$.
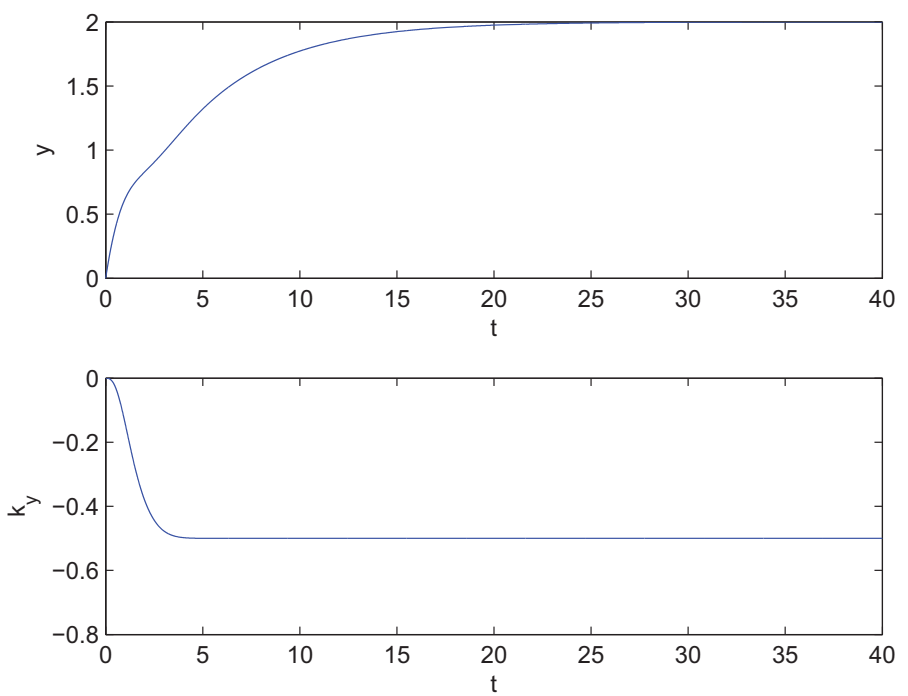

Fig. 9 - Closed-Loop Response of Non-Minimum Phase Plant 


\section{Conclusion}

This paper revisits the issue of adaptive control of linear uncertain systems with unmodeled dynamics by exploiting a feature of the optimal control modification adaptive law whereby the closed-loop system in the limit tends to a linear system asymptotically as the adaptive gain tends to infinity. For linear uncertain plants under certain conditions, the modification parameter $v$ has a limiting value. Taking the advantage of the asymptotic closed-loop system, a stability analysis can be performed to compute the modification parameter required to stabilize the closed-loop plant. This approach can be applied to linear uncertain systems with unmodeled dynamics, time delay, and non-minimum phase behaviors. The Rohrs counterexample is revisited using the optimal control modification adaptive laws. Utilizing the linear asymptotic property, the cross-over frequency and phase margin of the asymptotic closed-loop plant are derived analytically. By determining a suitable value of the modification parameter for a desired phase margin, the plant in the Rohrs counterexample is shown to be stabilized by the optimal control modification adaptive law. The linear asymptotic property is also used to design adaptive control for non-minimum phase plants with relative degree 1.

\section{References}

${ }^{1}$ Rohrs, C., Valavani, L., Athans, M., and Stein, G., "Robustness of Continuous-Time Adaptive Control Algorithms in the Presence of Unmodeled Dynamics," IEEE Transactions on Automatic Control, Vol AC-30, No. 9, pp. 881-889, 1985.

${ }^{2}$ Ioannou, P. and Kokotovic, P., "Instability Analysis and Improvement of Robustness of Adaptive Control," Automatica, Vol. 20, No. 5, pp. 583-594, 1984.

${ }^{3}$ Narendra, K. and Annaswamy, A., "A New Adaptive Law for Robust Adaptation Without Persistent Excitation,” IEEE Transactions on Automatic Control, Vol. AC-32, No. 2, pp. 134-145, 1987.

${ }^{4}$ Cao, C. and Hovakimyan, N., "Design and Analysis of a Novel $\mathscr{L}_{1}$ Adaptive Control Architecture with Guaranteed Transient Performance", IEEE Transactions on Automatic Control, Vol. 53, No. 2, pp. 586-591, 2008.

${ }^{5}$ Nguyen, N., Krishnakumar, K., and Boskovic, J., "An Optimal Control Modification to Model-Reference Adaptive Control for Fast Adaptation", AIAA Guidance, Navigation, and Control Conference, AIAA 2008-7283, 2008. (2012) pp. 485-494.

${ }^{6}$ Nguyen, N., "Optimal Control Modification for Robust Adaptive Control with Large Adaptive Gain," Systems \& Control Letters, 61

${ }^{7}$ Calise, A., Yucelen, T., Muse, J., and Yang, B., "A Loop Recovery Method for Adaptive Control," AIAA Guidance, Navigation, and Control Conference, AIAA-2009-5967, August 2009.

${ }^{8}$ Lavretsky, E., "Combined / Composite Model Reference Adaptive Control," IEEE Transactions on Automatic Control, Vol. 54 (11), pp. 2692-2697, 2009.

${ }^{9}$ Slotine, J.-J. and Li, W., Applied Nonlinear Control, Prentice-Hall, 1991. 